\title{
Sugar Palm Fibre-Reinforced Unsaturated Polyester Composite Interface Characterisation by Pull-Out Test.
}

\begin{abstract}
Polymer composites using natural fibres as the reinforcing agents have found their use in many applications. However, they do suffer from a few limitations, due to the hydrophilicity of the natural fibres which results in low compatibility with the hydrophobic polymer matrices. This paper aims to determine the best sugar palm (Arenga pinnata) fibre surface treatment to improve the fibre-matrix interfacial adhesion. Fibre surface modifications were carried out by water retting process where the fibres were immersed in sea water, pond water and sewage water for the period of 3 days. The test samples were fabricated by placing a single fibre in an unsaturated polyester resin. Single-fibre pull-out tests showed that freshwater-treated fibres possessed the highest interfacial shear strength, followed by untreated fibres, sewage water-treated fibres, and sea water-treated fibres. Further surface analyses of the samples were performed using a Scanning Electron Microscope (SEM) and an Energy Dispersive X-ray Spectroscopy (EDS) system. (2011) Trans Tech Publications.
\end{abstract}

Keyword: Composite; Sugar palm. 